\title{
Hovering Dual-Spin Vehicle Groundwork for Bias Momentum Sizing Validation Experiment
}

\author{
Paul M. Rothhaar*, Daniel D. Moerder ${ }^{\dagger}$ and Kyong B. Lim ${ }^{\ddagger}$ \\ NASA Langley Research Center, Hampton VA, 23681, USA
}

\begin{abstract}
Angular bias momentum offers significant stability augmentation for hovering flight vehicles. The reliance of the vehicle on thrust vectoring for agility and disturbance rejection is greatly reduced with significant levels of stored angular momentum in the system. A methodical procedure for bias momentum sizing has been developed in previous studies. This current study provides groundwork for experimental validation of that method using an experimental vehicle called the Dual-Spin Test Device, a thrust-levitated platform. Using measured data the vehicle's thrust vectoring units are modeled and a gust environment is designed and characterized. Control design is discussed. Preliminary experimental results of the vehicle constrained to three rotational degrees of freedom are compared to simulation for a case containing no bias momentum to validate the simulation. A simulation of a bias momentum dominant case is presented.
\end{abstract}

\section{Introduction}

Attitude stiffness is an attribute of a rigid body system's rotational dynamics in which the body resists rotation in one or more axes, and tends to restore the disturbed system to its original attitude. This attribute can be passively achieved by introducing a constant angular momentum bias into the system so that the body resists rotation along the directions normal to that of the bias momentum. When the bias is provided by an internal flywheel, so that the body is not required to rotate about its bias axis, we have a "dual spin" system.

Spacecraft designers have used dual spin for improving attitude performance for years, ${ }^{2}$ but the technique is also very attractive for aeronautical vehicles. In [1], and other studies, it has been seen that introducing a sufficiently large angular momentum bias along the vehicle's yaw axis results in a system that strongly resists and corrects deviations from trim in the pitch and roll axes. This passive attitude stiffness can substantially reduce the effort and bandwidth required of the vehicle's fluidic control effectors, such as thrusters, in the case of hovercraft, or trailing edge surfaces for fixed-wing vehicles.

This study focuses on validation of the methods discussed in reference [1]. This earlier paper provides expressions for sizing an angular momentum bias given a disturbance environment bandwidth and Power Spectral Density (PSD), and the desired Mean Square Response (MSR) of the vehicle Euler angles and body rates. The hardware, the Dual-Spin Test Device (DSTD) and related support equipment, was in a preliminary state in 2003 and was described in reference [3]. Now the hardware has matured and is ready for both experimental verification of bias momentum sizing expressions and experimental validation of bias momentum sizing methods. Unfortunately, due to some hardware failures, complete validation of bias momentum sizing is not offered in this publication. One test condition is offered and compared to simulation, however. Final results will appear in a publication at a later date.

The following material will introduce dual spin dynamics and review bias momentum sizing as described in reference [1] and the issues relevant to its validation. Descriptions of the DSTD hardware and mathematical models and related system are offered. Control laws are synthesized for the system over a range of stored angular momentum levels; comparability is discussed. Finally, the groundwork for the validation test is documented including preliminary results for some test conditions.

*Aerospace Technologist, Dynamic Systems and Control Branch, paul.m.rothhaar@nasa.gov

$\dagger$ †erospace Technologist, Dynamic Systems and Control Branch, daniel.d.moerder@nasa.gov

¥Aerospace Technologist, Dynamic Systems and Control Branch, kyong.b.lim@nasa.gov 


\section{Bias Momentum Sizing Overview}

A short summary of dual-spin dynamics and bias momentum sizing from reference [1] is presented for the sake of readability and to frame complications introduced by the DSTD hardware. The following first introduces the DSTD equations of motion with some simplifying assumptions. Then bias momentum sizing is summarized in general with some measures of system performance. Finally, a measure of the cumulative control activity is defined.

The full non-linear equations of motion represented in scalar form are written in terms of the body axes which are defined in figure 1. These are simplified by fixing the body axes to the vehicle cg. The resulting equations $(1,2,3)$ show coupling between all axes due to both inertial and gyric effects.

$$
\begin{gathered}
\tau_{1}=I_{1} \dot{\omega}_{1}+\omega_{2}\left[h_{B}+\omega_{3}\left(I_{3}-I_{2}\right)\right] \\
\tau_{2}=I_{2} \dot{\omega}_{2}+\omega_{1}\left[h_{B}+\omega_{3}\left(I_{3}-I_{1}\right)\right] \\
\tau_{3}=I_{3} \dot{\omega}_{3}+\dot{h}_{B}+\left(I_{2}-I_{1}\right) \omega_{1} \omega_{2}
\end{gathered}
$$

where $I$ is the vehicle's inertia, $h_{B}$ is the bias momentum, $\omega_{i}$ is the angular velocity of the $i^{\text {th }}$ axis, and $\tau_{i}$ is the torque on the $i^{\text {th }}$ axis.

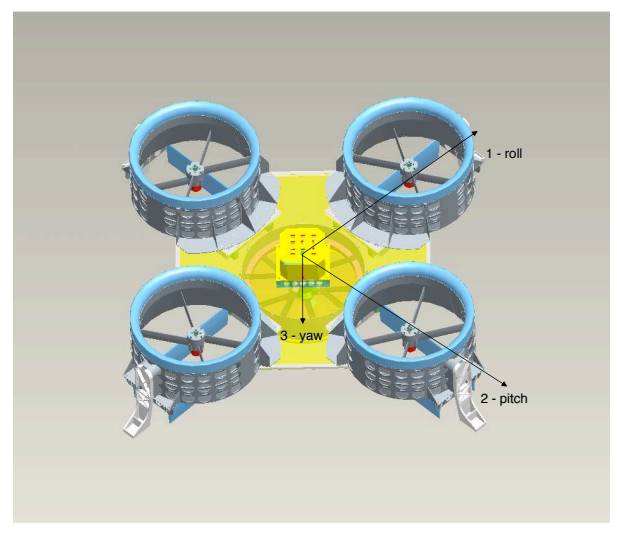

Figure 1. Dual-Spin Test Device CAD model with axes

Angular momentum products in each equation introduce nonlinearity to the system. Making a few assumptions yields more physical intuition and helpful simplification. First, we can assume the bias momentum to be sufficiently large such that the system is gyrically dominant, ${ }^{1}$ i.e.

$$
\left\|h_{B}\right\|>>\|J \omega\|
$$

Furthermore, $\dot{h}_{B}$ from Eq. 3 is assumed to be zero since the momentum wheel is regulated at a constant spin rate. The vehicle is assumed to have inertial symmetry about the roll and pitch axes eliminating the coupling in yaw. These assumptions yield a physically intuitive linear model of the dual spin system.

$$
\begin{array}{r}
I_{1} \dot{\omega}_{1} \approx-\omega_{2} h_{B}+\tau_{1} \\
I_{2} \dot{\omega}_{2} \approx \omega_{1} h_{B}+\tau_{2} \\
I_{3} \dot{\omega}_{3} \approx \tau_{3}
\end{array}
$$

Yaw-axis-oriented bias momentum dominance causes cross coupling of the pitch and roll axis. Control torques applied to the pitch axis produce effects in the roll axis, and vice versa. So, the pitch and roll axes are cross controlled in a gyroscopically dominant system as outlined in reference [7].

The bias momentum sizing approach in [1] relates the mean square response of the vehicle in pitch and roll to the bandwidth and variance of a stationary ergodic torque disturbance assumed to come from the

environment. For simplicity's sake, ${ }^{1}$ the disturbance is assumed to be independent in the roll and pitch axes, 
with constant power spectral density ( $S_{\tau_{1}}$ for roll, $S_{\tau_{2}}$ for pitch) over a common bandwidth $B W_{\tau}$. This gives an approximate mean-square torque disturbance

$$
\varepsilon\left(\tau^{T} \tau\right) \approx 2\left(S_{\tau_{1}}^{o}+S_{\tau_{2}}^{o}\right) B W_{\tau}
$$

where $\varepsilon$ denotes expectation. This leads to the sizing equations

$$
\begin{gathered}
\varepsilon\left[\omega^{T} \omega\right] \approx \frac{\varepsilon\left[\tau^{T} \tau\right]}{2 c I(B W)_{\tau}}\left[\arctan \left(\frac{x_{o}-1}{\zeta_{m}}\right)+\arctan \left(\frac{x_{o}+1}{\zeta_{m}}\right)\right] \\
x_{o} \triangleq \frac{B W_{\tau}}{\lambda_{o}} \approx \frac{B W_{\tau}}{\frac{h_{B}}{\sqrt{I_{1} I_{2}}}}
\end{gathered}
$$

where

c system viscous damping coefficient

$\zeta_{m} \quad$ viscous damping ratio

$I \quad$ vehical rotational inertia matrix

$\lambda_{o} \quad$ undamped precession frequency

$B W_{\tau}$ cutoff bandwidth of the disturbance torque

$h_{B}$ bias momentum

Plotting the mean square response for a particular disturbance environment yields some insight. ${ }^{1}$ If damping is small and $x_{o}<1$ then Eq. 9 becomes approximately

$$
\varepsilon\left[\omega^{T} \omega\right] \approx \frac{1}{h_{B}^{2}\left(1-x_{o}^{2}\right)} \varepsilon\left[\tau^{T} \tau\right]
$$

The key insight from equation 11 is that the mean square response varies with the inverse square of bias momentum. In summary, one should choose bias momentum high enough so the disturbance bandwidth is much lower than the precession frequency and the mean square response is small.

Bias momentum can reduce the required amount of high bandwidth active control of a hovering dual-spin system if the momentum bias is sufficiently large. Bias momentum cannot, however, mitigate persistent DC disturbances. Low bandwidth aerodynamic control is necessary to maintain trim. The Cumulative Control Activity (CCA) quantifies the cumulative amount of thrust vectoring used to maintain trim. The CCA is a sum of the squares of the propeller speeds $\left(\omega_{f a n_{i}}\right)$, and vane angles $\left(\theta_{v_{a n e}}\right)$ with respect to their trim values, $\hat{\omega}_{f_{a n}}$ and $\hat{\theta}_{\text {vane }_{j}}$, over time. The CCA can also be broken into the vane and propeller contributions $C C A_{\text {Vane }}$ and $C C A_{\text {Fanspeeds. }}$.

$$
\begin{gathered}
C C A(t)=\int_{0}^{t}\left(\sum_{i=1}^{4}\left(\omega_{\text {fan }_{i}}-\hat{\omega}_{\text {fan }_{i}}\right)^{2}+\sum_{j=1}^{8}\left(\theta_{\text {vane }_{j}}-\hat{\theta}_{\text {vane }_{j}}\right)^{2}\right) d t \\
C C A_{\text {Vanes }_{\text {and }}}(t)=\int_{0}^{t} \sum_{j=1}^{8}\left(\theta_{\text {vane }_{j}}-\hat{\theta}_{\text {vane }_{j}}\right)^{2} d t \\
C C A_{\text {Fanspeeds }}(t)=\int_{0}^{t} \sum_{i=1}^{4}\left(\omega_{\text {fan }_{i}}-\hat{\omega}_{\text {fan }_{i}}\right)^{2} d t
\end{gathered}
$$

\section{DSTD Hardware}

The Dual-Spin Test Device (see figure 1) is a VTOL thrust levitating platform equipped with a shrouded propeller thrust vectoring system and a momentum wheel that adds a significant angular momentum bias to the vehicle. For the purposes of this paper testing is performed on the vehicle in a gimbaled configuration where the vehicle is attached to a bearing that allows 3 degrees of rotational freedom. The vehicle contains 


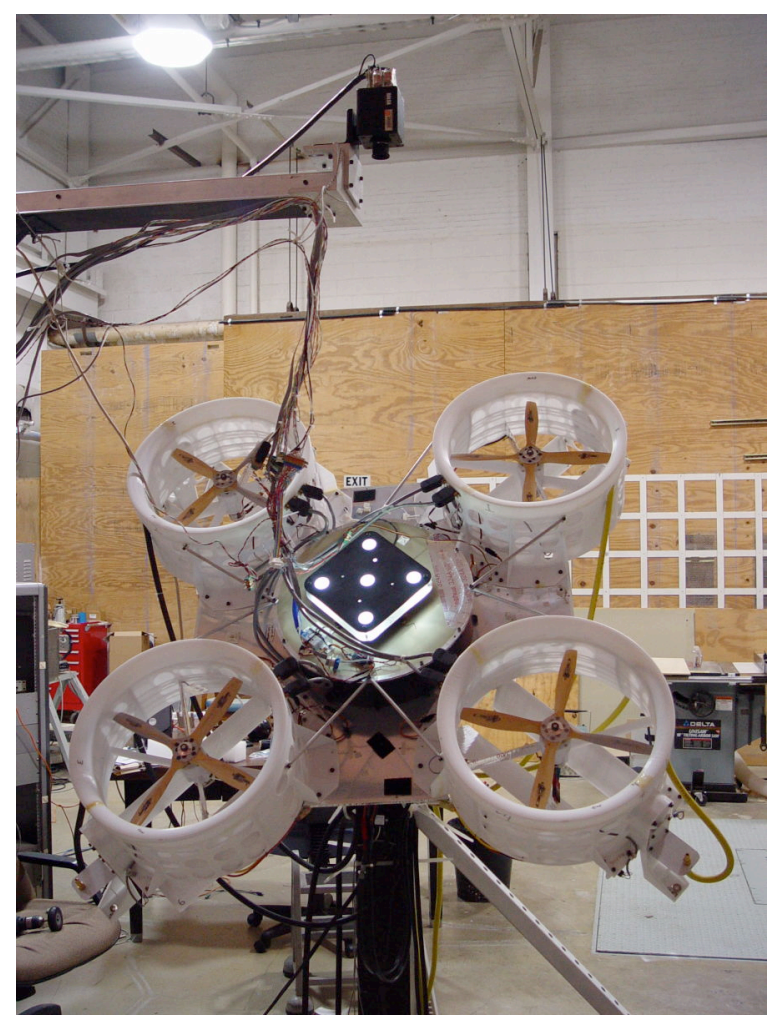

Figure 2. Dual-Spin Test Device Hardware and CAD Model With Axes

\begin{tabular}{|ccc|}
\hline Parameter & Symbol & Value \\
\hline Vehicle Mass & $\mathrm{m}$ & $18.15 \mathrm{~kg}$ \\
Vehicle Inertias & $I_{1}, I_{2}, I_{3}$ & $0.8,0.8,1.0\left(\mathrm{kgm}^{2}\right)$ \\
System damping & $\zeta$ & 0.1 \\
Length from cg to fan centerline & $l_{\text {cg } \rightarrow \text { fan }}$ & $0.4669 \mathrm{~m}$ \\
shroud inner diameter & $D_{\text {shroud }}$ & $0.3429 \mathrm{~m}$ \\
\hline
\end{tabular}

Table 1. Values for DSTD Parameters

several on-board and ground-based sensors for control and test measurements. Physical parameters including vehicle inertias, dimensions, component weights, and thrust vectoring data are included in table 1.

The thrust vectoring system diverts the shrouded propeller thrust vector via a pair of control vanes oriented radially from the vehicle center. The pair may operate symmetrically or asymmetrically. Symmetric and asymmetric vane angle along with differential thrust between fan units yield sufficiently large controllable torques about the pitch, roll, and yaw axes. The propellers are proportionally slaved to the one across the diagonal of the platform as in

$$
\omega_{f a n_{1}}+\omega_{f a n_{3}} \approx \omega_{f a n_{2}}+\omega_{f a n_{4}}
$$

This keeps the magnitude of the total angular momentum vector nearly constant during maneuvers. The propeller rotation directions are counter-rotating for any two shrouds on an edge of the platform. The shrouded propeller static data, polynomial models, and control allocation scheme are included in the Appendix.

The momentum wheel, shown in figure 3, provides an angular momentum bias proportional to its spin rate and rotational inertia. The momentum wheel is an aluminum spoke and rim design. A brushless DC motor drives the wheel within a safe operating range of approximately $0-4000 \mathrm{RPM}$. The wheels rotational inertia is $0.0411 \mathrm{~kg}-\mathrm{m}$ which yields up to $17 \mathrm{Nms}$ of angular momentum. This upper angular momentum 
limit is sufficient to maintain a bias momentum dominant state where the vehicle dynamics are dominated by gyric effects. ${ }^{1}$

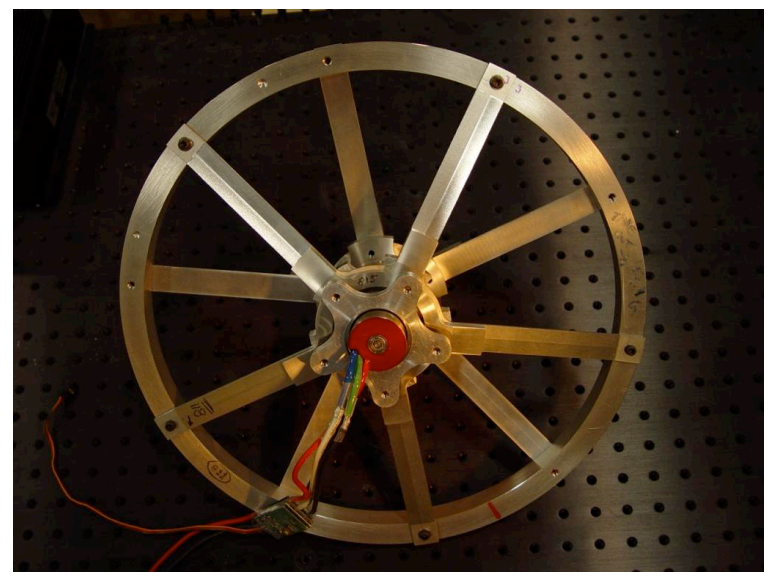

Figure 3. DSTD's momentum wheel

The DSTDs sensor suite measures the vehicle's fan speeds, vane angles, attitude, angular rates, position, and accelerations. The suite is divided between on-board and ground based sensors. On-board sensors include a Microbotics MIDG inertial measurement unit. It measures accelerations and angular rates on all three body axes. Hall effect sensors mounted on the shrouded propeller motors sense a magnet mounted on the motor shaft to yield propeller RPM. Potentiometers sense the vane positions. All on-board sensors provide data to the on-board PC104 computer running a Simulink ${ }^{\circledR}$ program compiled through Matlab ${ }^{\circledR}$ 's Real Time Workshop ${ }^{\circledR}$. A ground based vision system measures the vehicle position and attitude. This system includes a camera and a PC. The camera transmits images of a dot pattern mounted on the vehicle to dedicated hardware in the PC which calculates and subsequently outputs position and attitude. These measurements are transmitted to the on-board computer or a ground based data system at approximately $50 \mathrm{~Hz}$.

A $J R 3^{\circledR}$ load cell measures forces and moments on all three axes during non-flight static and dynamic thrust vectoring model parameter identification tests as well as some of the gimbaled bias momentum sizing tests. The load cell transmits data to dedicated hardware in a PC computer that is either recorded there or transmitted to the on-board computer system.

A gimbal joint (see figure 4) provides three degrees of rotational freedom. The attachment point is adjustable so that the DSTD may be mounted at or below the cg; some counterweights are necessary. The gimbal consists of a shaft through two bearings, providing yawing freedom, and a universal joint, providing roll and pitch freedom.

Four nozzles are situated vertically, one at the end of each shroud, (see figure 5). The nozzles are are connected by hoses to a bank of solenoid valves in a ground based system. These are supplied with compressed air. The valves can only be open or closed; there is no throttling. Forces are produced at the shroud tip when a solenoid opens. Since, the vehicle is gimbaled the forces are canceled at the rotation point. Only pure torques are applied by the system.

\section{Bias Momentum Sizing Validation Experiment Groundwork}

The section gives an overview of the groundwork for the bias momentum validation test. The DSTD propeller 2 motor burned up during testing. This prohibited the collection of data for any test conditions having stored angular momentum. An experiment was performed for the no-bias-momentum test condition. This experiment is compared to simulation. Good correlation between the experiment and simulation provide further validation of the simulation at the other test conditions as well, and thus to the concept of using bias momentum to augment vehicle stability. In place of measured data a simulation of the vehicle with 17.2 Nms of bias momentum is presented and compared to the simulation at 0 Nms of bias momentum.

The simulation results of the following sections are based on the DSTD mounted on its gimbal. This simulation contains non-linear equations of motion and the static and dynamic thrust vectoring system 


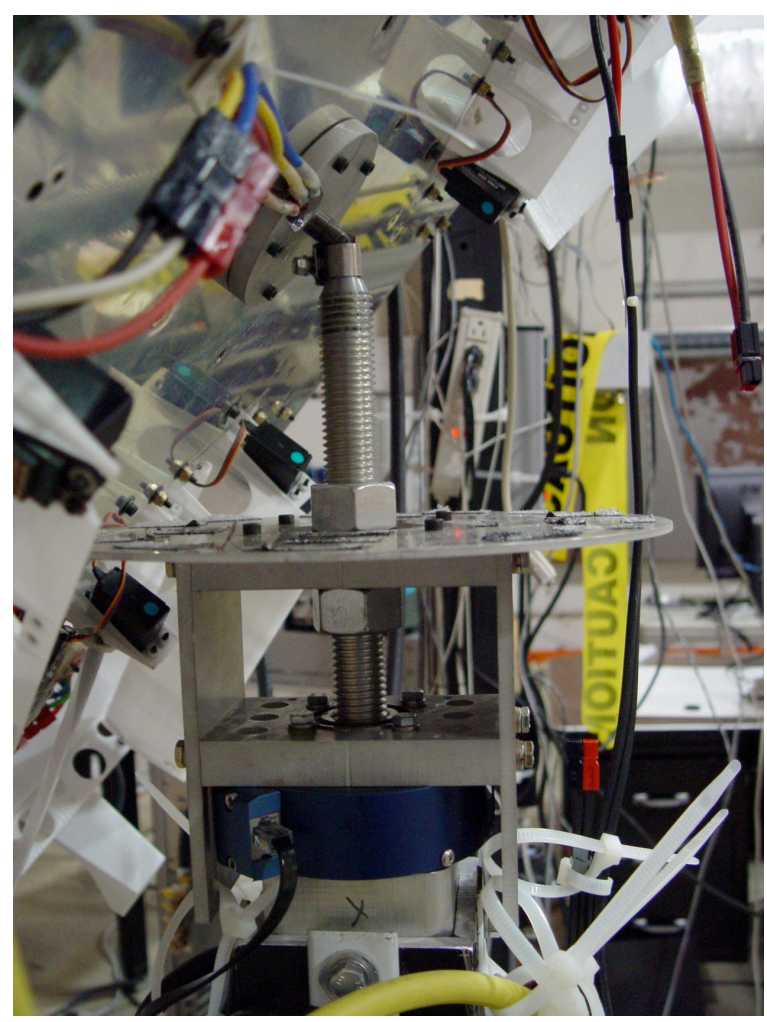

Figure 4. Dual-Spin Test Device gimbal joint

equation found in the appendix. The control law used for closed loop simulations is an LQR type and is described below.

Trade studies between mean square response of the vehicle and the bias momentum levels show bias momentum improves performance in certain regions of the frequency ratio $x_{o}$, (see equation 10). To experimentally verify these trades, bias momentum levels must be varied over a variety of disturbance bandwidths and the corresponding vehicle angular response must be measured. The frequency $x_{o}$ begins the process of selecting numerical values. This ratio separates the trade space into three important regions. If $x_{o}>1$ the precession frequency of the system dominates the mean square response. However, if $x_{o}<1$ the precession frequency is not excited by the disturbance. So, the mean square response is attenuated in proportion to the bias momentum level. Since this is the region most advantageous for stabilization we chose the maximum amount of bias momentum our momentum wheel can produce, approximately $17 \mathrm{Nms}$. The $B W_{\tau_{\max }}$ is then limited to ensure $x_{o}<1$.

A bench test of the disturbance system identifies the maximum variance of the disturbance torque at the $\max B W_{\tau}$. This yields the spectral densities $\left(S_{\tau_{1}}^{o}, S_{\tau_{2}}^{o}\right)$ of the torque disturbance about the roll and pitch axes. The gust disturbance system is driven by a random binary signal of known bandwidth. Each thruster receives it own unique random signal. The supply pressure varies in proportion to the number of jets on at any time. This results in a torque about the pitch and roll axes having a constant power spectral density up to $1.5 \mathrm{~Hz}$, the cutoff bandwidth. The PSD of this signal can be seen in figure 7 . The air jets create torque time histories, as measured by the JR3 load cell, on the vehicle that may be seen in figure 6 . The torque produced, seen in figure 7, has a nearly constant power until approximately $1.5 \mathrm{~Hz}$. A structural resonance of the vehicle exists at $10 \mathrm{~Hz}$. This can be seen in the PSD of the torque signal in figure 8 . The vehicle will need to be structurally reinforced before testing with bias momentum to avoid excitation of the precession frequency from structural vibrations.

The jet system may be run at different pressures and bandwidths. The result is a repeatable and scalable simulated gust environment of known power spectral density, bandwidth, and variance valid for static testing and hover conditions. 


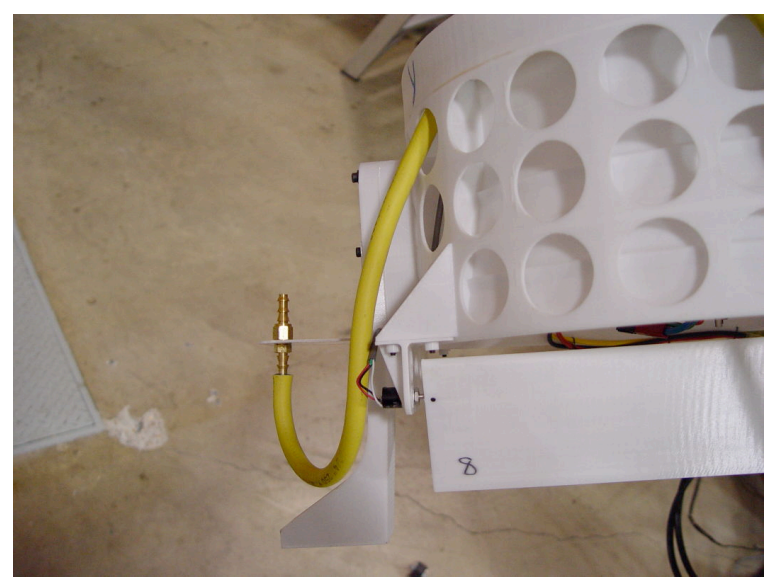

Figure 5. Gust simulation jet

\begin{tabular}{|ccc|}
\hline$\tau_{\text {Gust }}$ & $\operatorname{MSR}\left(N m^{2}\right)$ & $B W_{\tau}$ \\
\hline$\tau_{x}$ & 0.3141 & $\approx 1.5 \mathrm{~Hz}$ \\
$\tau_{y}$ & 0.2335 & $\approx 1.5 \mathrm{~Hz}$ \\
$\tau_{z}$ & 0.0590 & $\approx 1.5 \mathrm{~Hz}$ \\
\hline
\end{tabular}

Table 2. Disturbance Torque Sizing Parameters
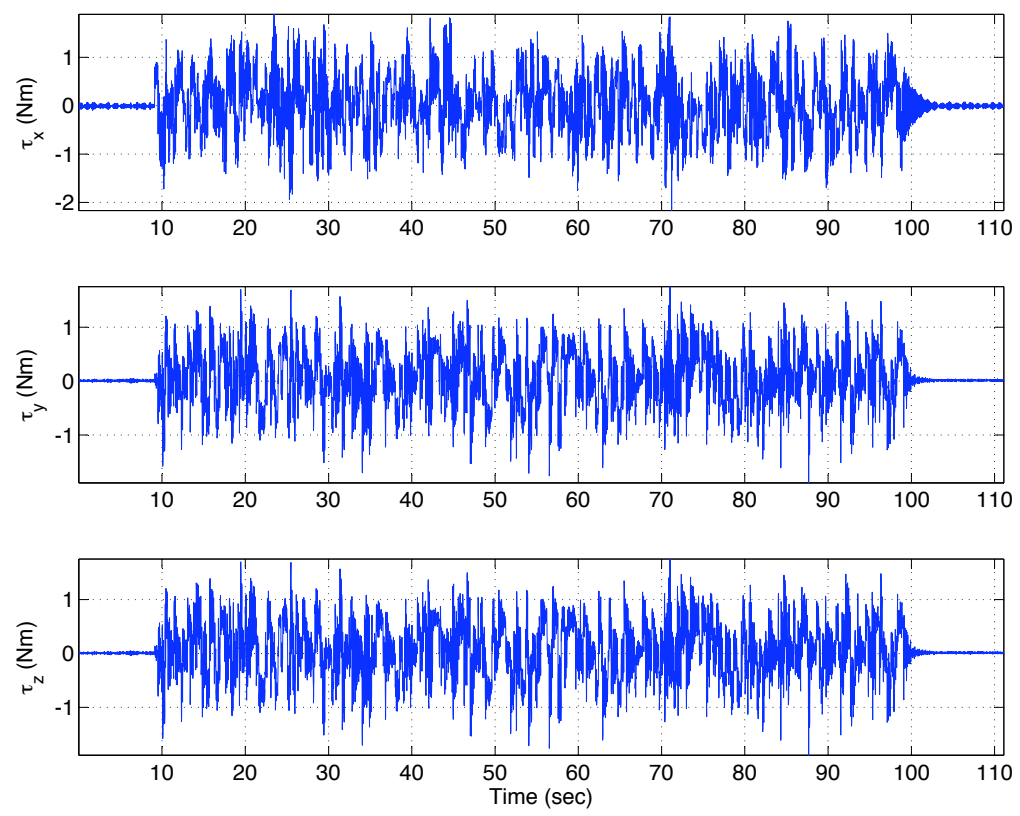

Figure 6. Torque time history of gust disturbance 


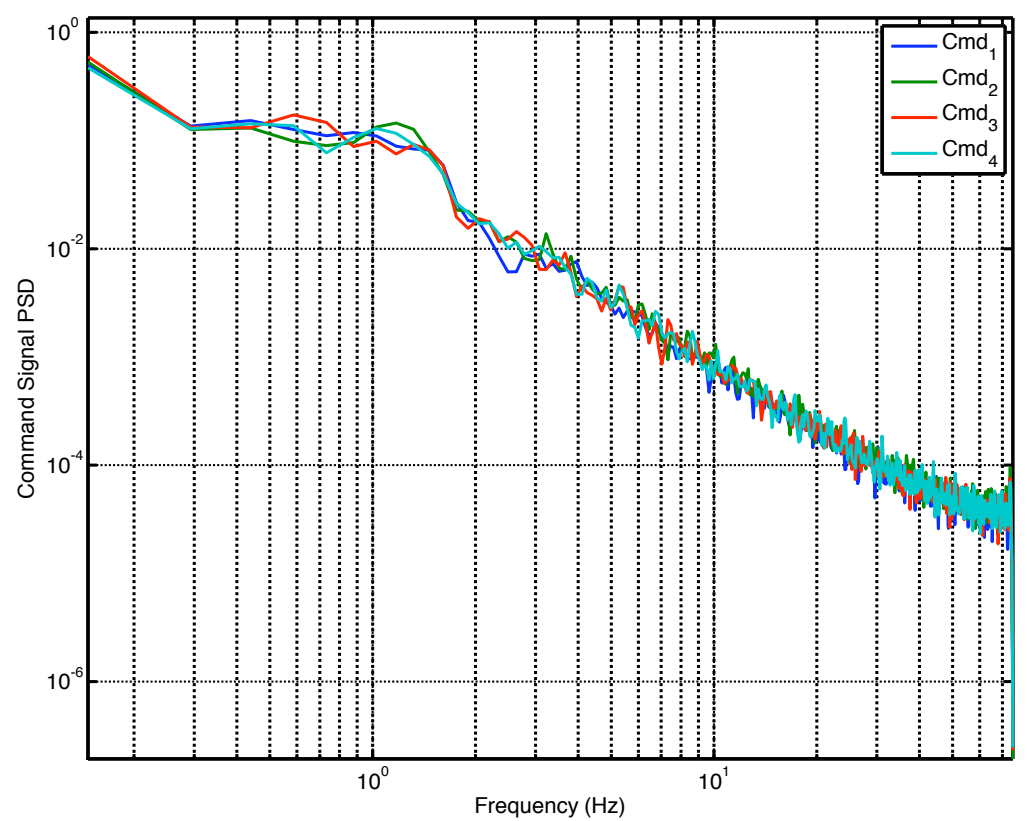

Figure 7. Power Spectral Density of air jet signal

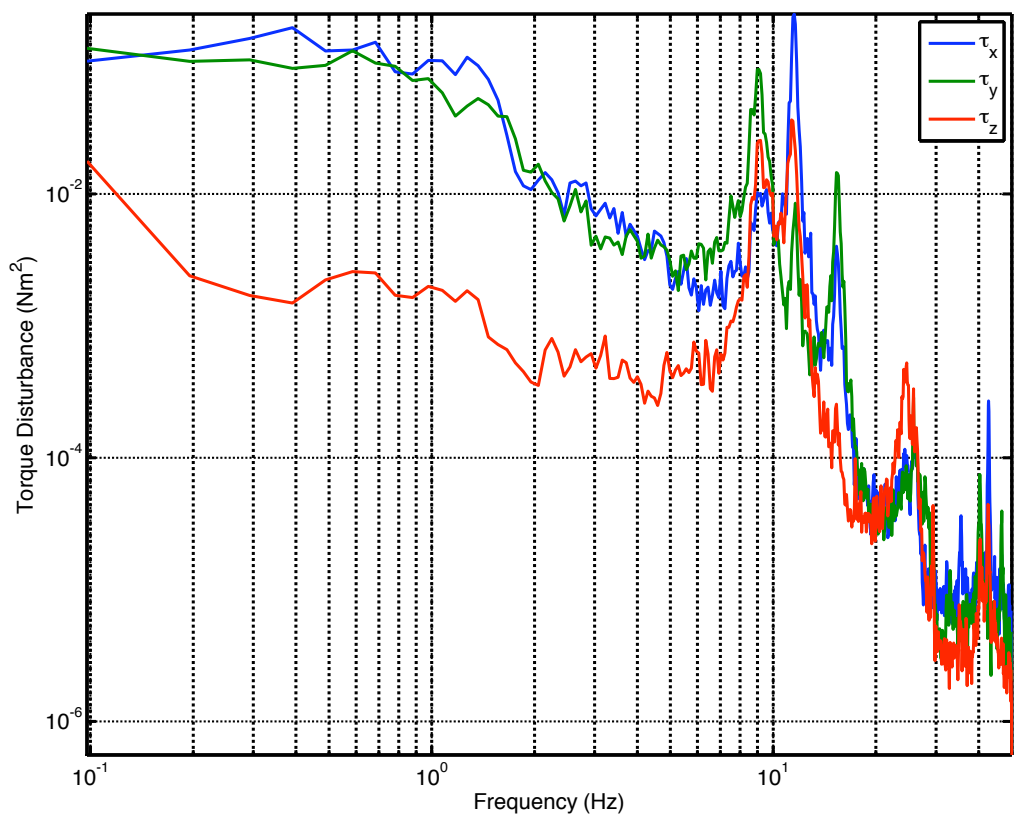

Figure 8. Power Spectral Density of gust disturbance torque

8 of 19 
A multi-variable Linear Quadratic Regulator (LQR) control posed as a tracking problem is used to return the vehicles attitude to a trimmed state or track an attitude command. The controller is synthesized using nine states including three angular velocities, three Euler angles and the integrated error between the Euler angles and the trim value for all three axes. Control is synthesized using the same LQR weights for each bias momentum level to ensure fair comparison. The control effort is defined as the CCA found in equation 12. The cost for the LQR problem is in terms of the attitude errors $\left(\beta_{i}\right)$, the vehicle body rates $\left(\omega_{i}\right)$, and the integral error of the attitude angles $\left(\gamma_{i}\right)$ is

$$
J(t)=\int_{0}^{t} \sum_{i=1}^{3}\left(\left(\beta_{i}-\hat{\beta}_{i}\right)^{2}+\left(\omega_{i}-\hat{\omega}_{i}\right)^{2}+\left(\gamma_{i}-\hat{\gamma}_{i}\right)^{2}\right) d t
$$

where $\hat{\omega}_{i}, \hat{\gamma}_{i}$, and $\hat{\beta}_{i}$ denote trim values for each parameter.

The linear DSTD model $(5,6,7)$ is used for control design. Physical quantities may be found in the appendix. The trim condition is straight and level, meaning that the attitude angles are all set to zero. The lifting force produced is less than that necessary to lift the platform which reduces wear on the hardware and provides a more relaxed testing environment.

\subsection{Experiment and Simulation Results}

At this point only one experimental test condition was performed. The test is run with no bias momentum in the system. The following figures $(9,10,3,11)$ compare the simulated test with the measured data. The same torque disturbance is applied to both the simulation and the DSTD. Close correlation between the simulation and the experiment validates the simulation.

The first two plots, in figures 9 and 10, show the Euler angles and rates for both the simulation and DSTD experiment. The simulation predicts the DSTD's Mean Square Response (MSR) of the pitch and roll attitude angles to within $5.6 \%$ and the vehicle body rates to within $1.9 \%$, (see tables 3 and 4 ). The yaw angle does not correlate as well between the simulation and experiment. The control allocation algorithm assumes that the yaw command for the vane angles may be superimposed over the asymmetric commands. The larger, yet still small, error here is probably a symptom of this assumption when the vanes are not operating in their linear region. This assumption may need to be revisited if large angle maneuvers are attempted.

The power spectral density of the simulated and experimental cases attitude angles in figure 11 have a relatively flat power until $0.2 \mathrm{~Hz}$ where they drop off slowly till $1.5 \mathrm{~Hz}$. They drop off rapidly at this point since the disturbance at this frequency rolls off rapidly as well, as seen figure 8. The Cumulative Control Activity of the vanes, seen in figure 12, shows that the simulation and experiment agree to within $7.3 \%$ at the worst point. The $C C A_{\text {Fanspeeds }}$ agrees poorly being off by a factor of 3.5 believed to be caused by excessive sensor noise in the measured data and some fan model errors in the simulation. This plot has three separate pairs of curves. The largest curve is the total CCA found in equation 12. The smaller curves are the contributions of the vanes and the propeller speeds to the total CCA.

\begin{tabular}{|ccc|}
\hline Euler Angles & $M S R_{E x p}\left(D e g^{2}\right)$ & $M S R_{\text {Sim }}\left(D e g^{2}\right)$ \\
\hline Roll & 1.865 & 1.660 \\
Pitch & 1.317 & 1.710 \\
Yaw & 0.557 & 0.032 \\
\hline Veh. Body Rates & $M S R_{E x p}\left(\frac{D e g}{\sec }\right)^{2}$ & $M S R_{\text {Sim }}\left(\frac{D e g}{s e c}\right)^{2}$ \\
\hline Roll & 13.028 & 13.727 \\
Pitch & 13.189 & 12.977 \\
Yaw & 1.096 & 0.245 \\
\hline
\end{tabular}

Table 3. Mean Square Response of Euler angles and vehicle rates for experimental and simulated data 

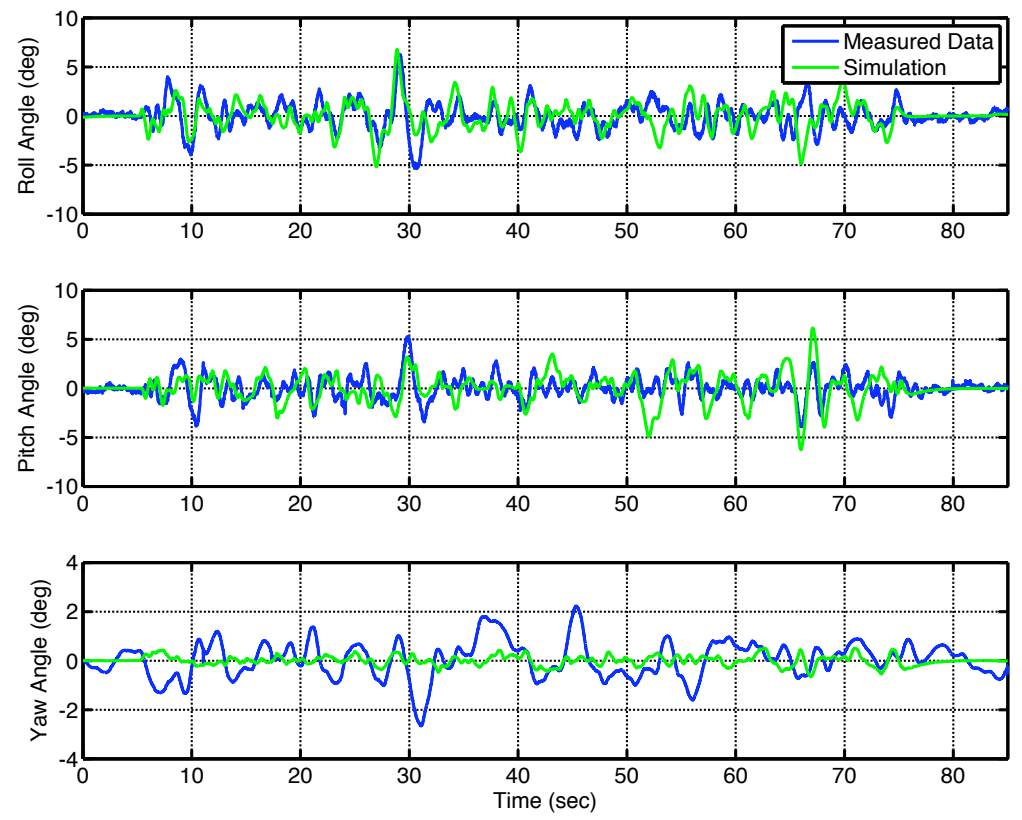

Figure 9. Time history of Euler angles for simulation and experiment
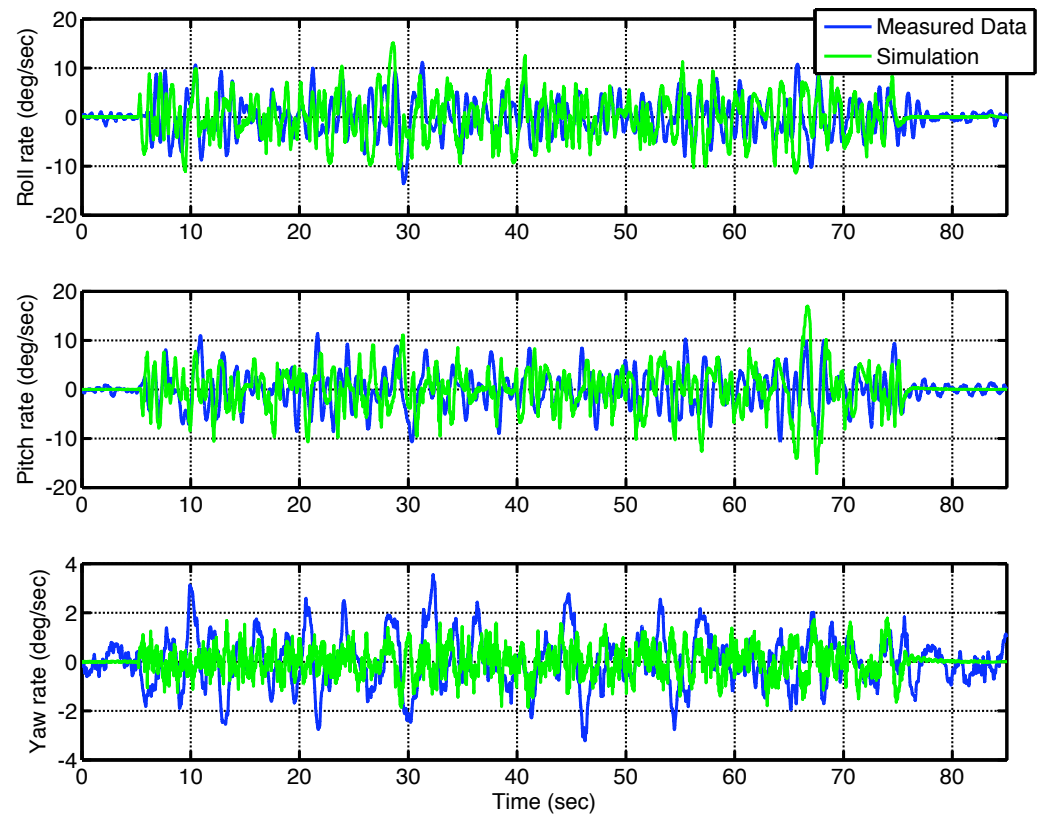

Figure 10. Vehicle attitude rates for simulation and experiment 


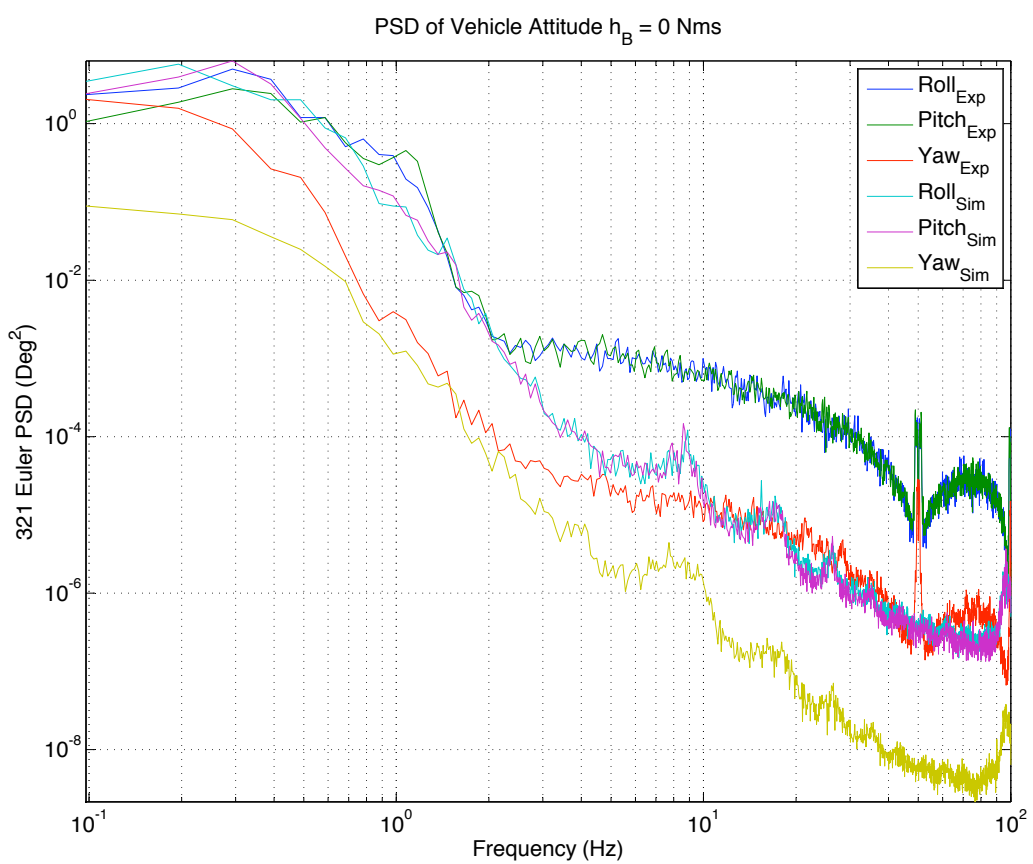

Figure 11. PSD of Euler Angles for simulation and experiment

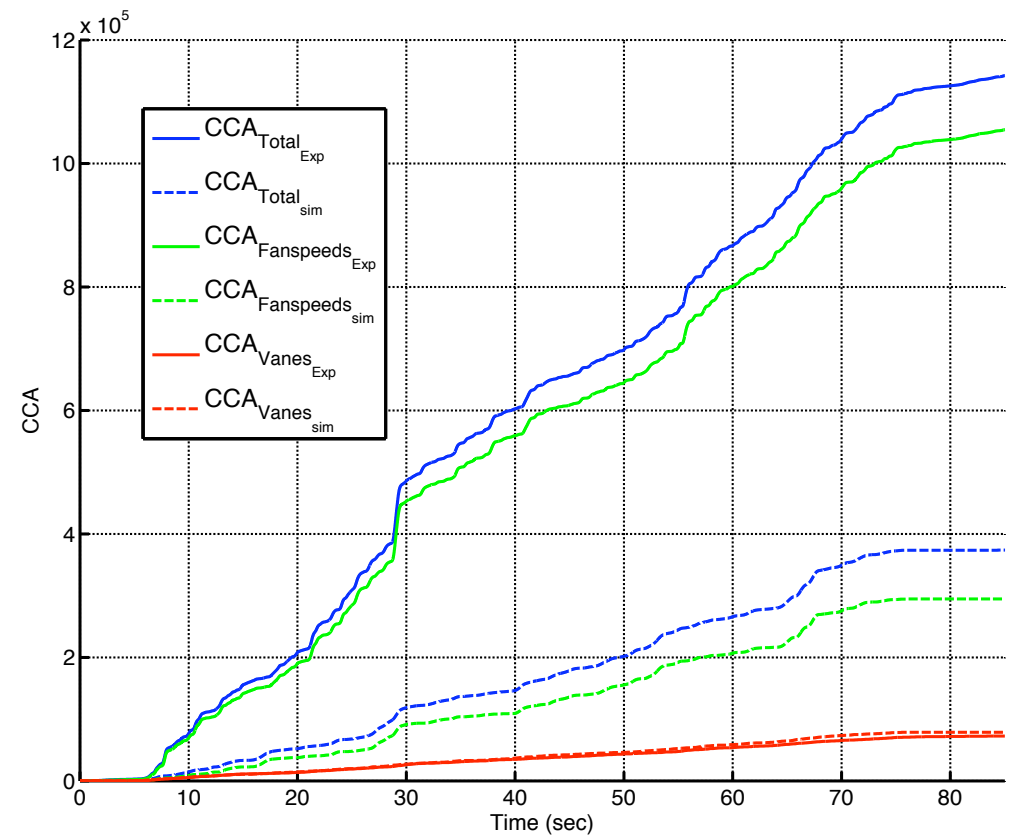

Figure 12. Cumulative Control Activity for simulation and experiment 


\subsection{Simulation Results for Large Bias Momentum Level}

Due to significant hardware failures on the DSTD the results for cases containing bias momentum cannot be presented until repairs are made. Instead, the advantages of bias momentum dominated systems are reiterated here with the current simulation.

The following plots show the previous simulation of the DSTD under the disturbance environment in figure 6 with $h_{B}=0 \mathrm{Nms}$. The maximum level of $h_{B}=17 \mathrm{Nms}$ is also included. The PSD seen in figure 15 shows the spectral power for the pitch and roll of the $h_{B}=17 \mathrm{Nms}$ case is far smaller than the $h_{B}=0 \mathrm{Nms}$ case over the low bandwidth region. The high bias momentum simulation maintains trim with no increase in control activity. The MSR,(table 4), of the attitude and rates shows an order of magnitude improvement over the no bias momentum case. The CCA in figure shows no increase between the two cases.
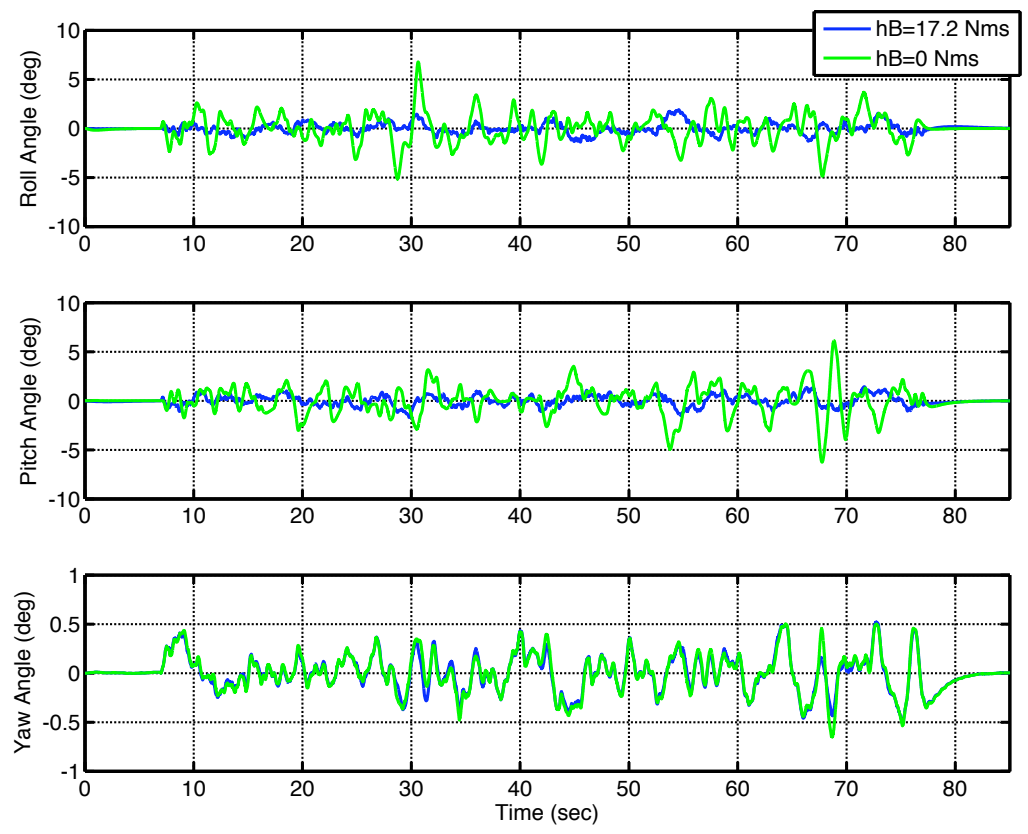

Figure 13. Euler attitude angles for simulated experiment between bias momentum levels

\begin{tabular}{|ccc|}
\hline Euler Angles & $M S R_{h B=17 N m s}\left(D e g^{2}\right)$ & $M S R_{h B=0 N m s}\left(D e g^{2}\right)$ \\
\hline Roll & 0.223 & 1.629 \\
Pitch & 0.222 & 1.677 \\
Yaw & 0.029 & 0.031 \\
\hline Veh. Body Rates & $M S R_{h B=17 N m s}\left(\frac{D e g}{s e c}\right)^{2}$ & $M S R_{h B=0 N m s}\left(\frac{D e g}{s e c}\right)^{2}$ \\
\hline Roll & 3.479 & 13.463 \\
Pitch & 3.725 & 13.727 \\
Yaw & 0.2398 & 0.239 \\
\hline
\end{tabular}

Table 4. Mean Square Response of Euler angles and vehicle body rates for no bias momentum and large bias momentum 

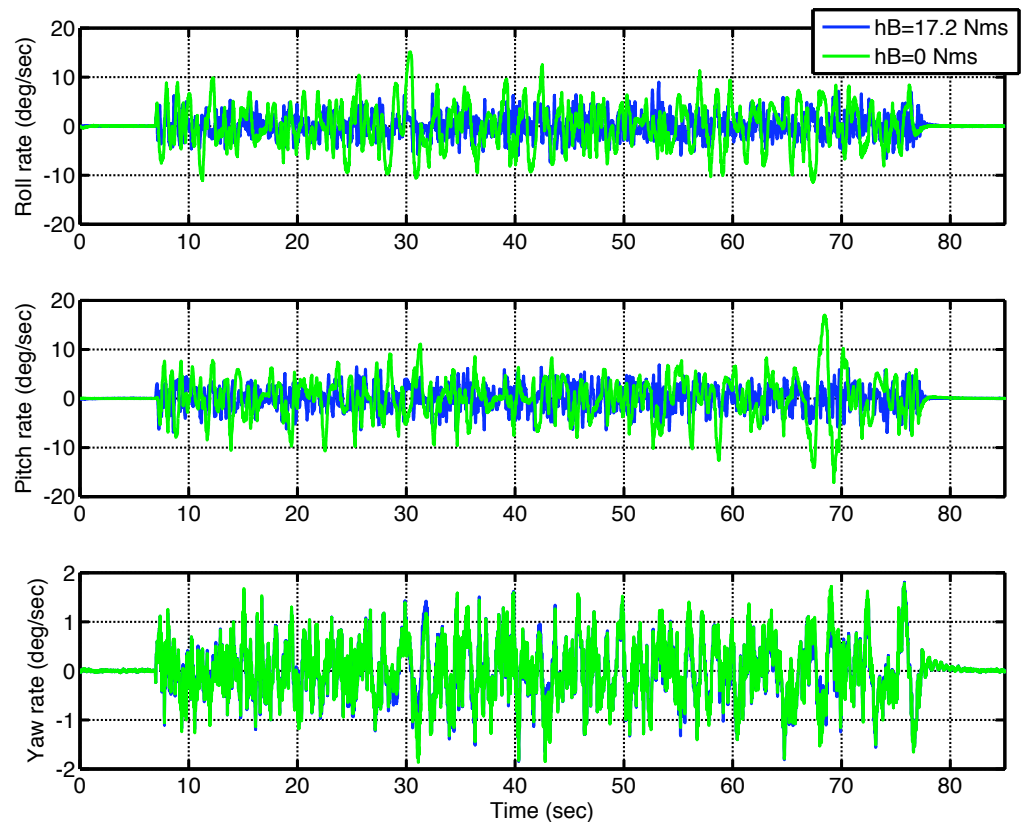

Figure 14. Vehicle body rates for simulated experiment between bias momentum levels

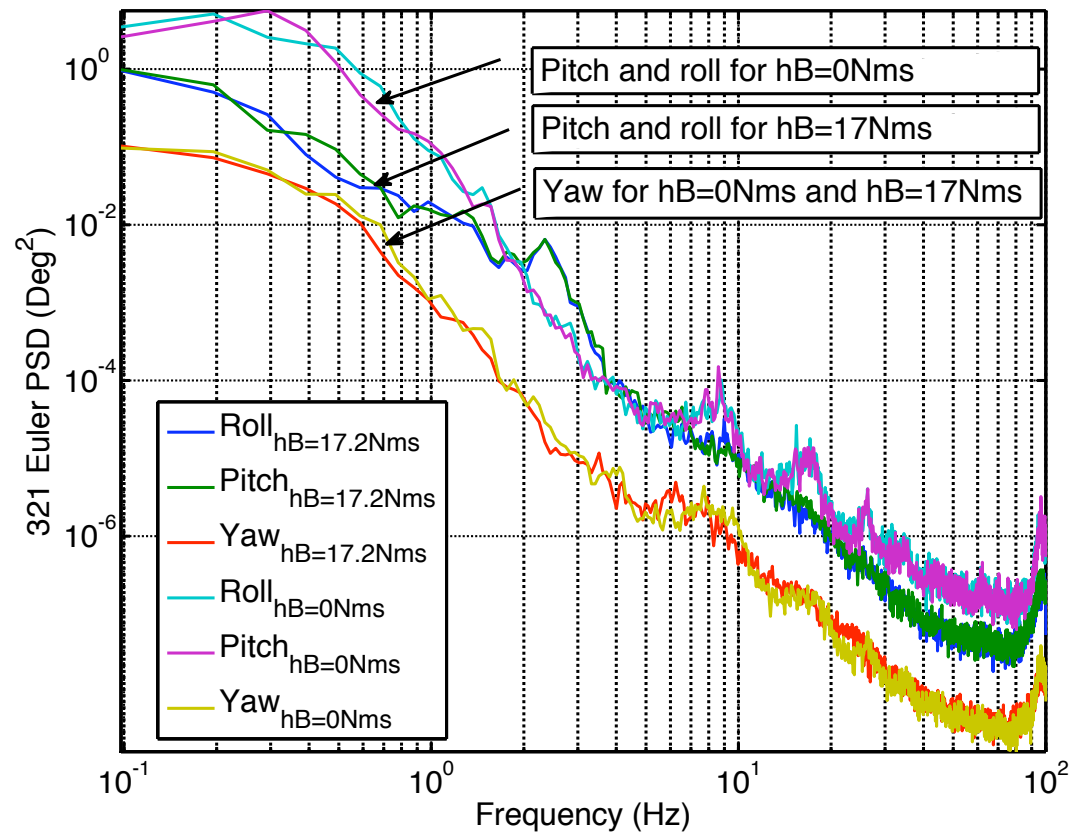

Figure 15. PSD for simulated experiment between bias momentum levels 


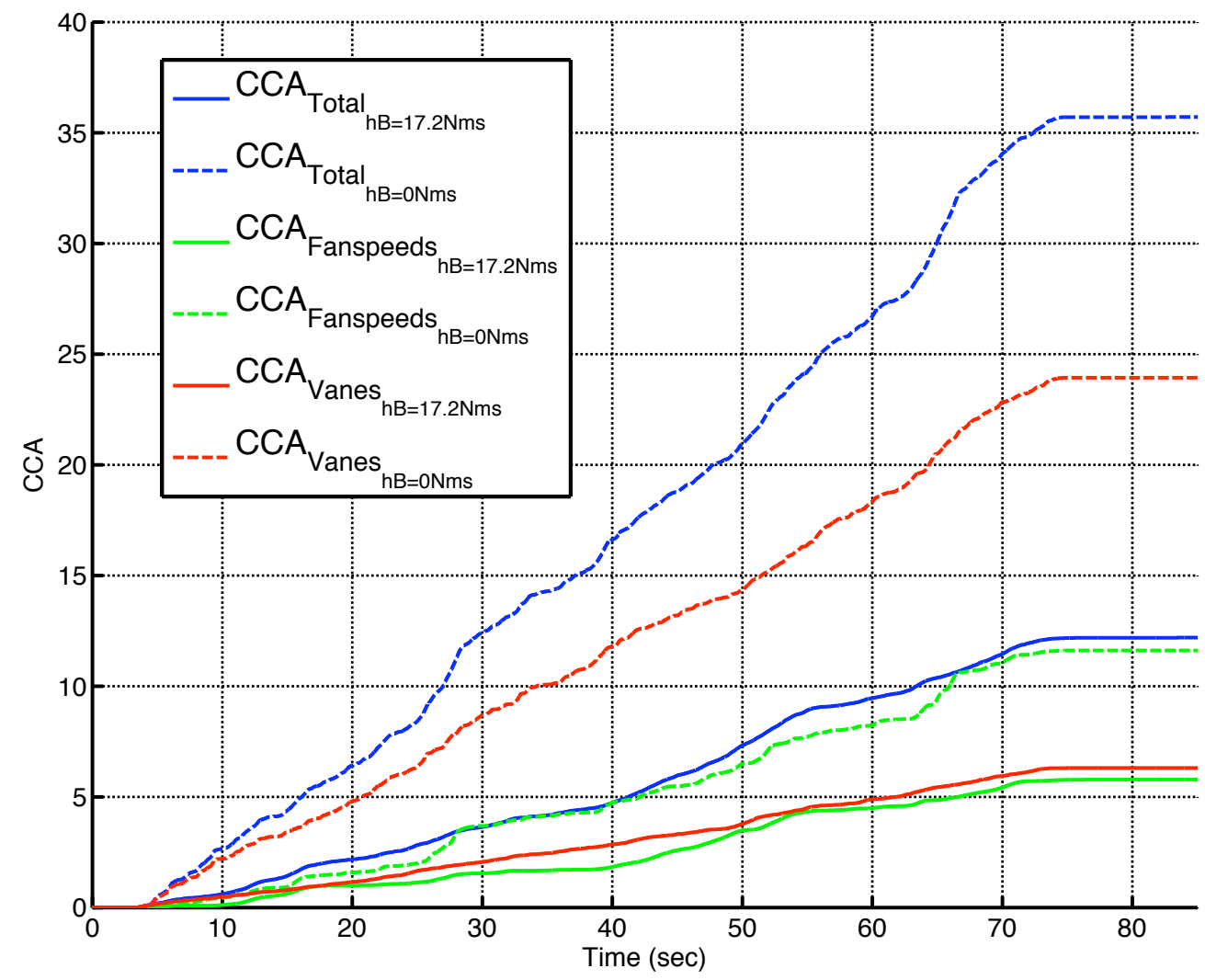

Figure 16. CCA for simulated experiment between bias momentum levels 


\section{Conclusions}

Limited validation of bias momentum sizing for dual spin systems was presented. An experiment was conducted that applied a known disturbance to the Dual-Spin Test Device. This disturbance has known and reproducible cutoff bandwidth and spectral power. The vehicle's Mean Square Response and Cumulative Control Activity were measured and compared to simulation. A PSD of the vehicle's attitude response showed attenuation by the controller and the bandwidth cutoff of the disturbance environment. The simulation's PSD, Mean Square Response, and Cumulative Control Activity agree closely to experimental values in the pitch and roll axes.

A simulation was run of the Dual-Spin Test Device under a bias momentum dominant condition and showed the value of bias momentum for reducing the vehicle's inherent reaction to gusts. Confidence in the simulation at this test condition is strengthened considering the agreement between the simulation and experiment at the no bias momentum test condition. A future experiment of the bias momentum dominant test conditions is suggested. 


\section{Appendix}

\section{A. DSTD Thrust Vectoring Model}

The thrust vectoring model relates fan speeds and vane angles to net propulsive forces and torques on the vehicle. Static bench test data was used to generate an experimentally verified polynomial model of the system. The data presented here is tailored for producing pure torques for attitude control.

\section{A.0.1. Symmetric Vane Configuration Static Data}

Symmetric vane configurations direct the vane trailing edges of a shroud in the same direction. These are used to create yaw torques. A bench test is run to identify the ability of the shrouds to create torques about the vehicle yaw axis. Steady state data is taken oven the operating range of the shrouds. Figure 17 below shows the measured data and a polynomial model fit to this data.

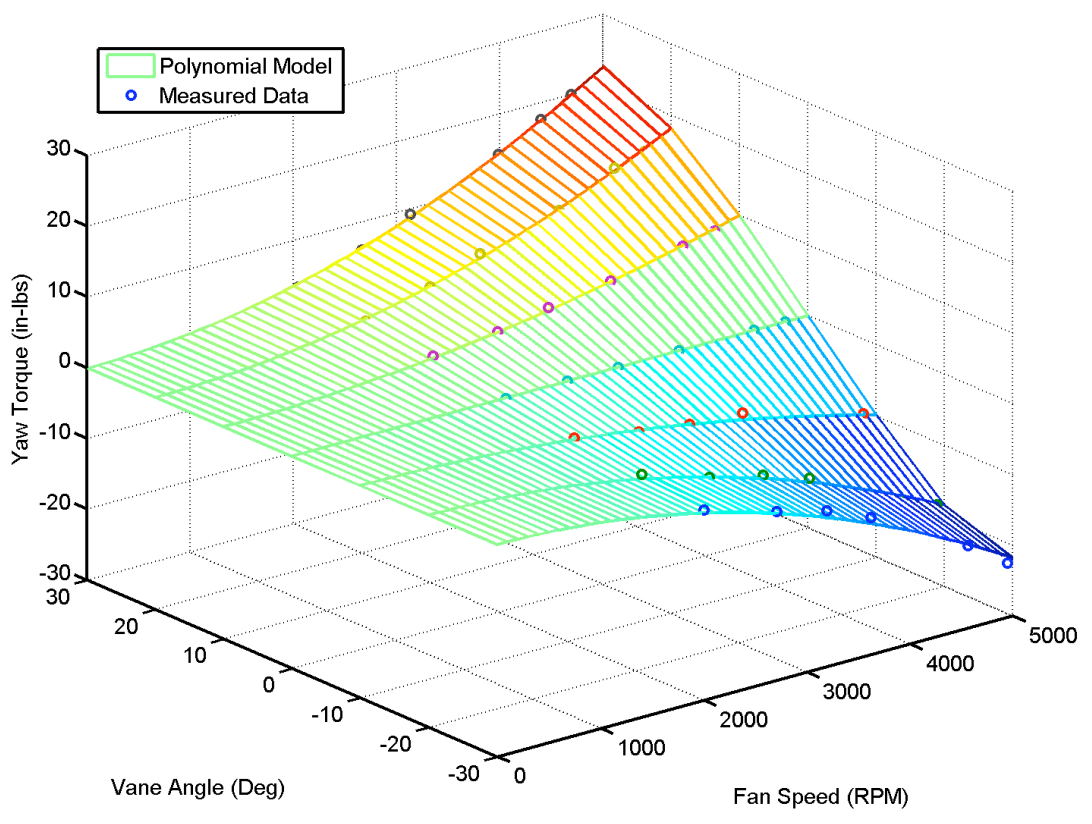

Figure 17. Yaw torque as a function of vane angle and fan speed

\section{A.0.2. Asymmetric Vane Configuration Static Data}

Asymmetric vane configurations occlude the flow through the shrouds by directing the vane trailing edges either toward or away from each other on a shroud unit. Occluding the flow on one shroud produces a thrust differential between it and the opposing shroud. This allows for pitch and roll torque creation. In figure 18 the change in thrust on a shroud is shown. This data is a polynomial fit to bench test data plotted in 19. The polynomial fit in 18 is the data found in figure 19 with the value of the zero vane angle data subtracted from the other data points at every propeller speed. In this way the change of thrust from asymmetric vane angles is known instead of the thrust value itself. In this model $\theta>0$ indicates vane trailing edges are rotated inward by the amount of theta, likewise $\theta<0$ indicates vanes rotating away at angle $\theta$. 


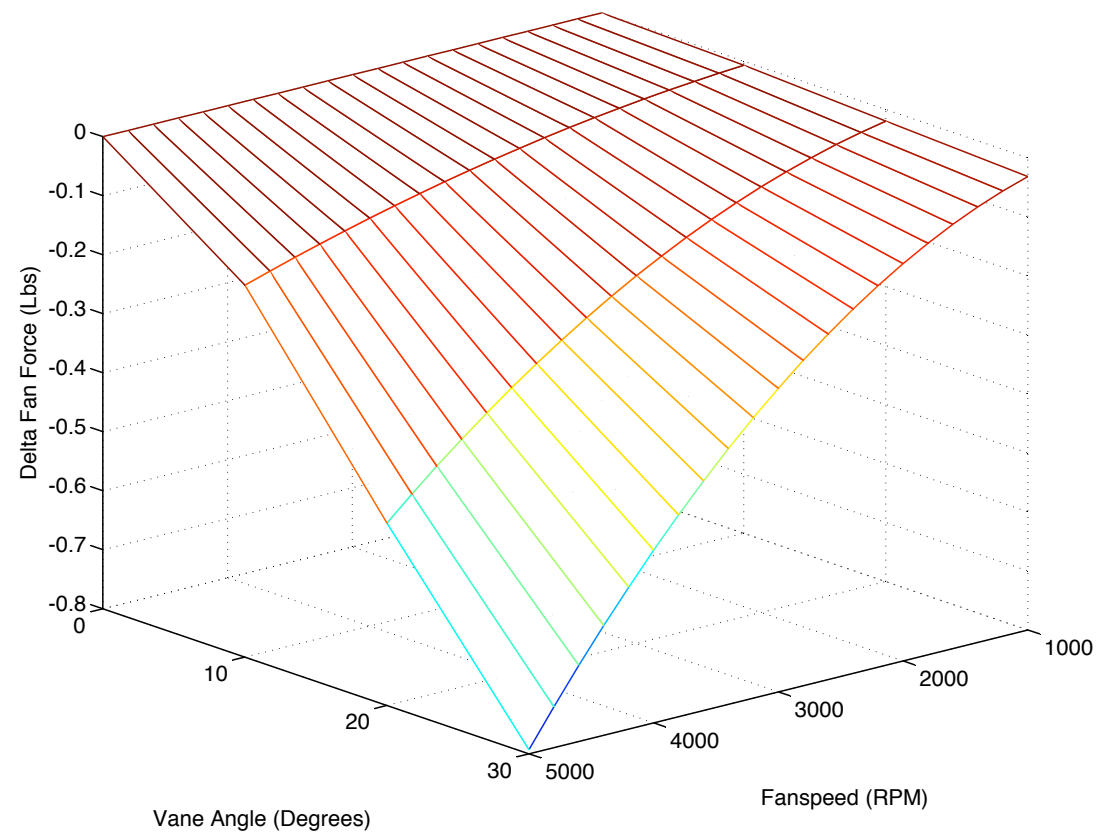

Figure 18. Change in thrust using asymmetric vane configurations

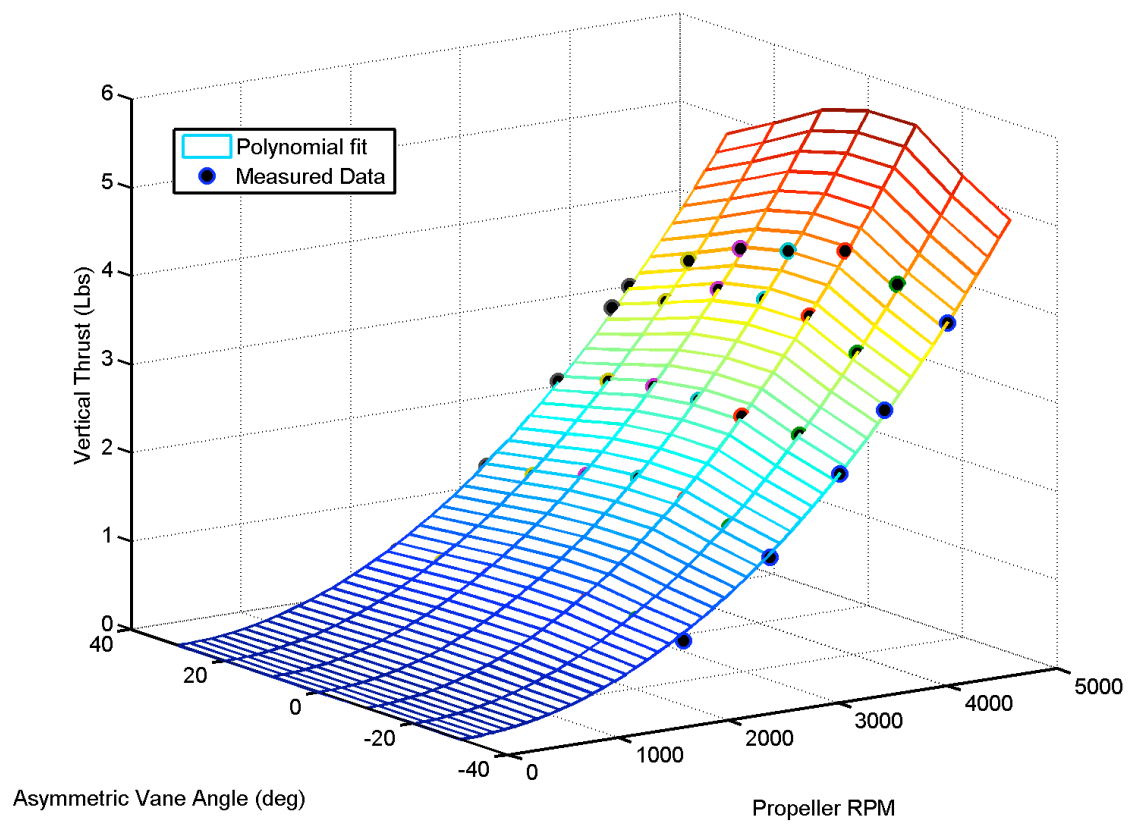

Figure 19. Change in thrust using asymmetric vane configurations 


\section{A.0.3. Fan Thrust and Differential Thrusting}

Opposing shrouded propeller units may also create pitch and roll torques about the vehicle cg by differential thrusting. A bench test was performed over a range of fan RPM to identify a nominal thrust model for a single propeller unit. Figure [20] shows the measured data and a second order polynomial model.

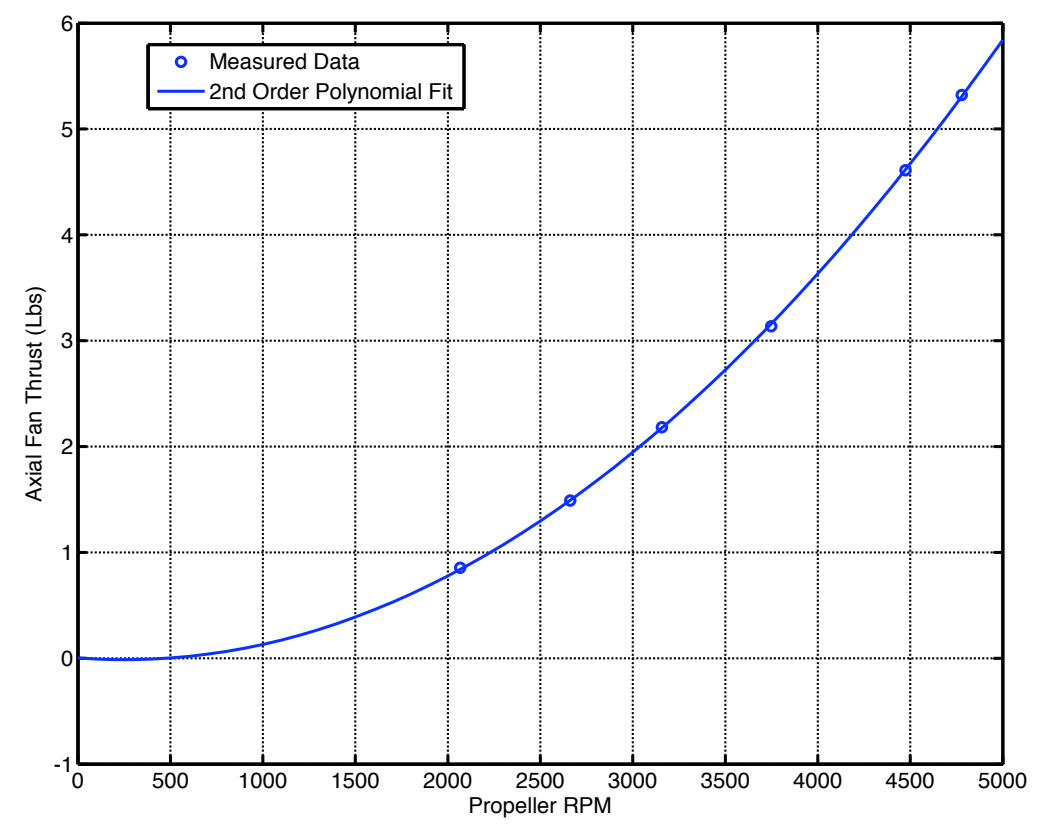

Figure 20. Shrouded propeller thrust over nominal range of RPM values

\section{A.0.4. Control Allocation Configuration}

Combinations of symmetric and asymmetric vane angles, and differential propeller thrusting provide controllable torques about all three axes at two different bandwidths. The control vanes can operate with significantly higher bandwidth than the propeller motors. High frequency torque commands are sent to the inverse vane models via a high pass filter. The remaining low frequency content is sent to the differential thrusting inverse models. The bandwidth of the propellers and vanes are determined via dynamic tests the nominal operating conditions. The vanes are limited to a bandwidth of $10 \mathrm{~Hz}$ at the nominal thrust condition where the propellers run at 4500 RPM. Both the vane and motor dynamics are assumed to be of the first order. The transfer function found from measured data and used in simulation to follow.

$$
\begin{aligned}
& T F_{\text {Vanes }}=\frac{1}{0.02 s+1} \\
& T F_{\text {Motors }}=\frac{1}{0.1 s+1}
\end{aligned}
$$




\section{References}

[1] Lim,K.B., Shin,J.Y., and Moerder, D.D., Bias momentum sizing for hovering dual-spin platforms, NASA Technical Paper 2006-214317, 2006.

[2] Ed.-Wertz, J.R., Spacecraft Attitude Determination and Control, Kluwer Academic Publisher, Boston, p. 787.

[3] Lim, K.B., Shin, J-Y, Cooper, E.G., Moerder, D.D., Khong, T.H., Smith, M.F, An Overview of The NASA Flying Test Platform Research, AIAA 2003-5775, 2003.

[4] Hughes, P.C., Spacecraft Attitude Dynamics, John Wiley \& Sons, Inc., New York, 1986.

[5] Newland, D.E., Random Vibrations and Spectral Analysis, Longman, London, 1981.

[6] Junkins, J.L., and Turner, J.D., Optimal Spacecraft Rotational Maneuvers, Elsevier, Amsterdam, 1986.

[7] Lim,K.B., and Moerder,D.D., Control Laws for a Dual-Spin Stabilized Platform, AIAA Guidance, Navigation and Control Conference, AIAA-2008-6484, AIAA Washington, DC, 2008. 